\title{
Distale Femurfrakturen extraartikulär und intraartikulär
}

Frank Graef, Serafim Tsitsilonis, Sven Märdian

\section{Einleitung}

Distale Femurfrakturen sind seltene, aber zumeist komplexe Verletzungen, die bei der Versorgung schwierige Anforderungen an das behandelnde Team stellen und mit einer hohen Komplikationsrate behaftet sind [1]. Nach der Quadrantenregel kann das distale Femur als ein Quadrat mit der Kantenlänge des größten Epiphysendurchmessers definiert werden [2]. Nach Schatzker und Lambert werden Frakturen bis $15 \mathrm{~cm}$ proximal der Kniegelenksebene zu distalen Femurfrakturen gezählt [3]. Die Inzidenz distaler Femurfrakturen wird in der Literatur mit 8,7/100000 Einwohner/Jahr angegeben [4]. Sie stellen 3\% der Femurfrakturen und 0,4\% aller Frakturen dar und gehören damit zu den selteneren Verletzungen [5]. Hinsichtlich der Geschlechterverteilung sind Frauen häufiger betroffen (Verhältnis 2:1). Es zeigt sich ein bimodales Verteilungsmuster bezüglich des Lebensalters zum Zeitpunkt der Verletzung: Junge Patienten erleiden diese Frakturen zumeist im Rahmen von Hochrasanz-Traumata oder Sportunfällen, ältere Patienten sind von Niedrigenergie-Traumata betroffen [4]. Eine aktuelle epidemiologische Studie konnte zeigen, dass 65\% der distalen Femurfrakturen ab dem 60. Lebensjahr auftreten und es sich damit um eine Fraktur von vornehmlich älteren Patienten handelt [4]. In der klinischen Praxis hat sich die AO/OTA-Klassifikation (Arbeitsgemeinschaft für Osteosynthesefragen, Orthopaedic Trauma Association) zur Einteilung dieser Frakturen durchgesetzt. In der Mehrzahl der Fälle handelt es sich um extraartikuläre (Typ A) oder partiell-intraartikuläre (Typ B) Frakturen. Die Häufigkeit von vollständig intraartikulären Typ-C-Verletzungen am distalen Femur wird mit ca. 6\% angegeben [4]. Bei der klinischen und radiologischen Diagnostik ist die Überprüfung des neurovaskulären Status, aufgrund des häufig nach posterior abgekippten Frakturfragments, obligat. Hierbei können dorsal verlaufende Strukturen wie die Aa. femoralis et poplitea oder die Nn. ischiadicus et tibialis kompromittiert werden. Goldstandard in der Therapie von distalen Femurfrakturen ist die operative Stabilisierung. Die konservative Therapie dieser Verletzungen ist Patienten vorbehalten, deren Gesundheitszustand eine operative Therapie nicht zulässt oder welche die operative Intervention ablehnen. [6]. Trotz moderner, hochstandardisierter OP-Techniken kommt es nach wie vor in ca. $14 \%$ der Fälle zu postoperativen Komplikationen wie Pseudarthrosen, Infektionen oder Pneumonien [1]. Die vorliegende Arbeit gibt eine Übersicht über die angewandte Anatomie, Diagnostik, Therapie und Nachbehandlung extra- und intraartikulärer Femurfrakturen.

\section{Anatomische Besonderheiten}

Ziel der rekonstruktiven Chirurgie bei der Behandlung von Frakturen ist u.a. die anatomische Rekonstruktion der Gelenkfläche mit stabiler Fixierung, sodass eine frühfunktionelle Nachbehandlung möglich ist. Neben der muskulären und neurovaskulären Topografie ist die genaue Kenntnis der knöchernen Anatomie Grundvoraussetzung einer erfolgreichen Operation. Um Abweichungen der Rotations- oder Achsverhältnisse bei einem bestimmten Patienten festzustellen, kann die Überprüfung der kontralateralen Seite, z. B. mittels Bildverstärker (BV) intraoperativ, notwendig sein. Dadurch können auch etwaige Fehlstellungen postoperativ vermieden werden.

Das Femur gehört zu den langen Röhrenknochen mit zylinderförmigem Querschnitt. In der axialen Ansicht verlaufen die Kortizes dorsal spitz aufeinander zu, wodurch der Oberschenkelknochen im Querschnitt die Form eines Tropfens bekommt. Im Bereich des distalen Femurs verbreitert sich der Knochen sowohl im anterioposterioren als auch mediolateralen Durchmesser und bildet mit der medialen und lateralen Femurkondyle den femoralen Anteil des Kniegelenks. Die Kondylen des Femurs sind dorsal breiter als ventral. Dadurch bekommt der femorale Gelenkblock (vom Patientenende aus betrachtet) die Form eines Trapezoids, welches sich von dorsal nach ventral verjüngt. Die Verjüngung bzw. Inklination beträgt lateral ca. $15^{\circ}$ und medial ca. $25^{\circ}$. Diese Besonderheit ist im a.p. Röntgen nicht zu sehen, weswegen von lateral platzierte Schrauben - obwohl im a.-p. Röntgenbild nicht zu erkennen - zu lang sein können und dementsprechend medial zu Weichteilirritationen führen können $[8,9]$. Um die korrekte Lage der Schrauben und Platte mittels BV zu überprüfen, ist das Bein für die laterale Implantatlage nach außen und für die mediale Implantatlage entsprechend nach innen zu rotieren ( $\bullet$ Abb. 1 ). Zwischen beiden Kondylen befindet sich eine knöcherne Aussparung, die interkondyläre „Notch“. Die Begrenzung der Notch entspricht im konventionellen Röntgen der „Blumensaat-Linie“ ( $\mathbf{A b b .}$ 2) [7]. 

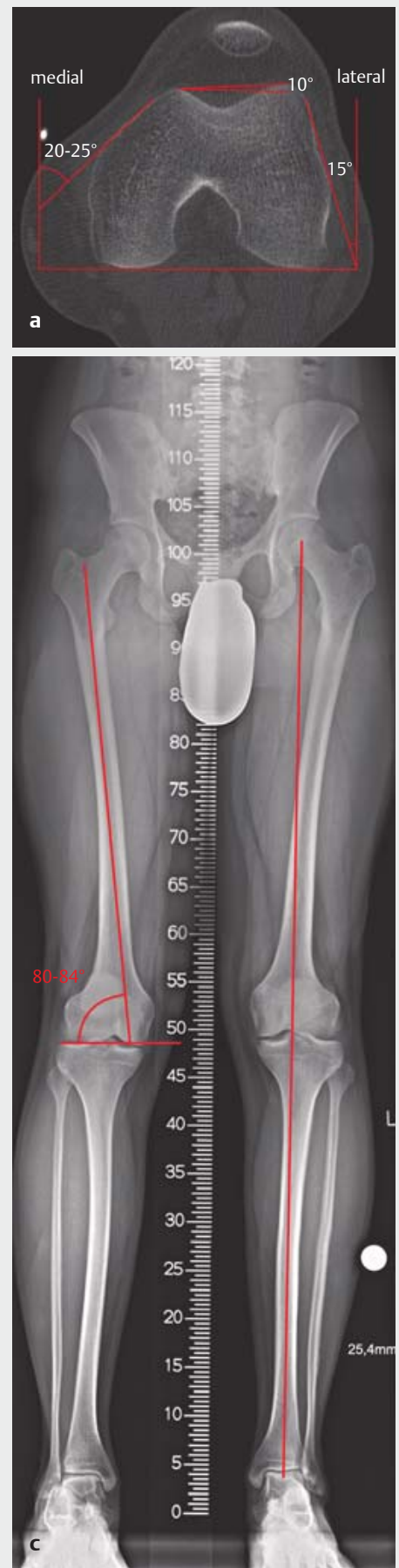

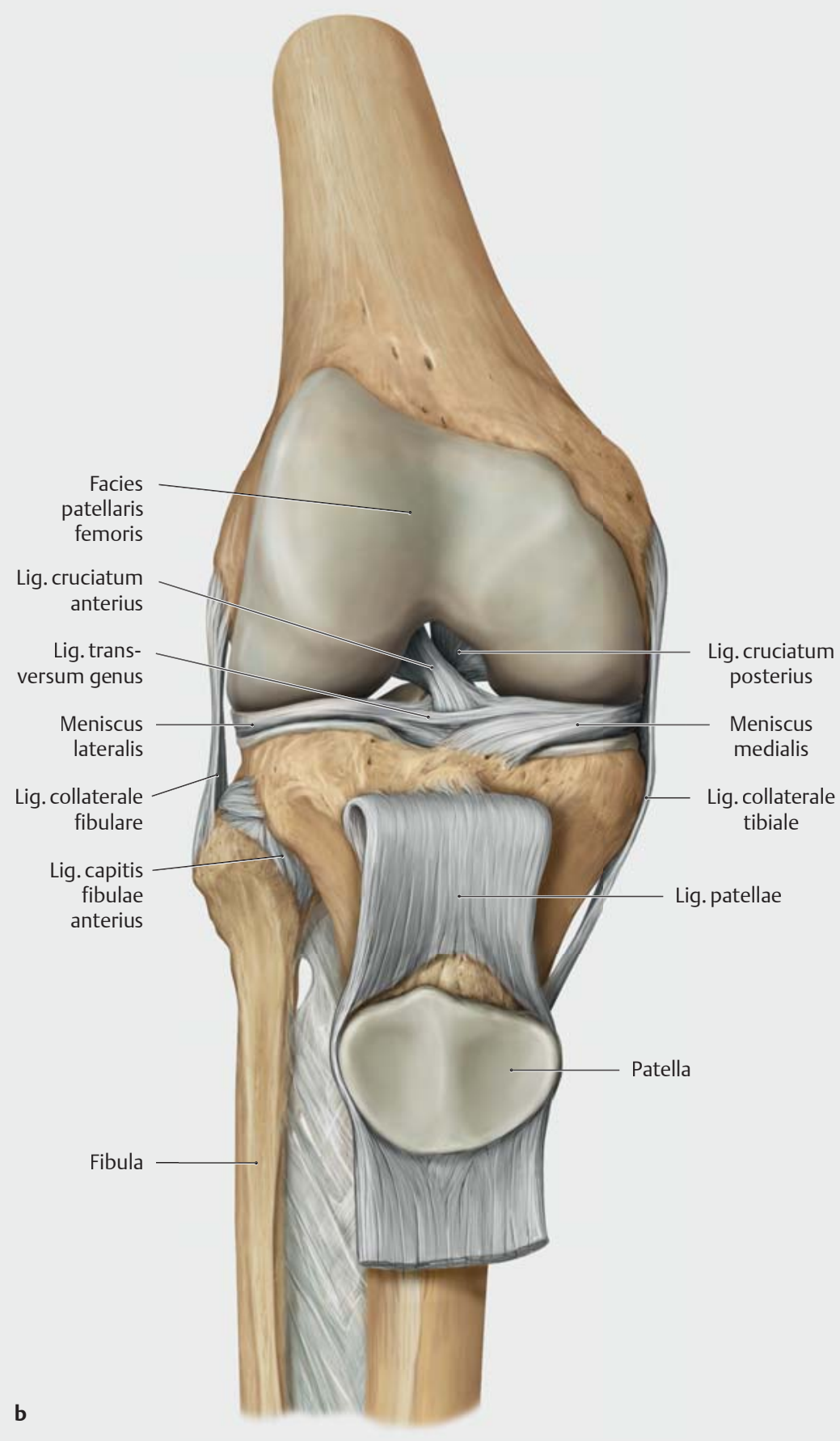

- Abb. 1 a Vom Patientenende aus betrachtet ist die posteriore Femurkondyle breiter als anterior, das distale Femur hat somit eine trapezoide Form, was im a.-p. Röntgen nicht zu sehen ist. Wird dieser Umstand missachtet, können Schrauben- und Plattenfehllagen die Folge sein. Die korrekte Implantatlage kann durch Innen- und Außenrotation des Beins festgestellt werden. b Bei der Instrumentierung von intraartikulären Frakturen ist zu beachten, dass die laterale femorale Knorpelfläche anterior länger ist als medial (Bildquelle [13]). Quelle: Schünke M, Schulte E, Schumacher U et al. 1.18 Brustbein und Rippen. In: Schünke M, Schulte E, Schumacher U et al., Hrsg. Prometheus LernAtlas - Allgemeine Anatomie und Bewegungssystem. 4. Aufl. Stuttgart: Thieme; 2014. doi:10.1055/b-004-129726. c Die mechanische Achse des Beins verläuft vom Zentrum des Hüftkopfes zum Zentrum des OSG und trifft das Knie ca. 5-10 mm medial des Kniegelenkszentrums, was einem physiologischen Varus von 1,2 ${ }^{\circ}$ entspricht. Die anatomische Achse des Femurs besitzt einen physiologischen Valgus von ca. $6^{\circ}$. 
Die Tragachse des Beins verläuft vom Hüftkopfzentrum bis zum Zentrum des oberen Sprunggelenks und trifft das Kniegelenk ca. 5-10 mm medial des Kniegelenkszentrums, was zu einem physiologischen Varus von ca. 1,2 führt [10]. Die anatomische Achse des Femurs hingegen steht mit ca. $6^{\circ}$ valgisch $[10,11]$. Die Gelenkfläche des Knies verläuft horizontal und parallel zur OSG-Gelenksebene, was intraoperativ ebenfalls überprüft werden sollte. Dabei wird das Kniegelenk bei der Kontrolle via Bildwandler ca. $10^{\circ}$ gebeugt, um den tibialen Slope auszugleichen [12]. Weiter ist bei der Instrumentierung am distalen Femur zu beachten, dass die Gelenkfläche des patellofemoralen Gleitlagers lateral länger ist als medial $(\triangleright$ Abb. 1).

Bei der Reposition der Fraktur sind die muskulären Kräfte zu beachten, durch die es letztlich zu der typischen Fehlstellung mit Innenrotation, Varus und Dislokation nach posterior kommt. Insbesondere durch den M. gastrocnemius kommt es zu einer Verkippung des distalen Frakturfragments nach dorsal mit einer resultierenden Retroversionsstellung. Durch den Zug des M. adductor magnus mit Ansatz am Tuberculum adductorium des medialen Epicondylus kann es zu einer Varusfehlstellung mit entsprechender Innenrotation und weiteren Verkürzung kommen [8].

\section{Diagnostik und \\ Therapieentscheidung}

Bei der Erhebung der Anamnese werden Nebenerkrankungen zur Einschätzung von Komorbiditäten ebenso wie der Unfallmechanismus erfragt. Dies kann erste Hinweise auf die Frakturlokalisation und -morphologie geben. Der Frakturmechanismus ist häufig die Kombination aus axialer Belastung mit Valgus,- Varus- oder Rotationskräften. Bei der Inspektion können die Patienten mit dem typischen klinischen Bild mit gebeugtem Knie auffallen, welches durch den Zug des M. gastrocnemius mit Verkürzung entsteht. Sowohl bei Hochrasanz-Traumata jüngerer Patienten als auch bei Niedrigenergie-Traumata älterer Patienten ist ein gründlicher Bodycheck im Rahmen des $\mathrm{ATLS}^{\circledR}$-Algorithmus obligat, um Begleitverletzungen auszuschließen. Differenzialdiagnostisch sind Verletzungen der Binnenstrukturen des Kniegelenks wie eingeschlagene Meniskusrupturen mit Extensionsdefizit im Kniegelenk, Bandläsionen oder Frakturen der Patella und der proximalen Tibia in Erwägung zu ziehen [14-16].

Beim klinischen Verdacht auf eine distale Femurfraktur ist zunächst ein konventionelles Röntgenbild in 2 Ebenen des Kniegelenks indiziert. Entsprechend der Frakturmorphologie kann es zudem notwendig sein, das gesamte Femur mit abzubilden. Weitere Röntgenaufnahmen des Beckens, der Hüfte oder weiterer Lokalisationen können beim Hinweis auf weitere Verletzungen notwendig sein.

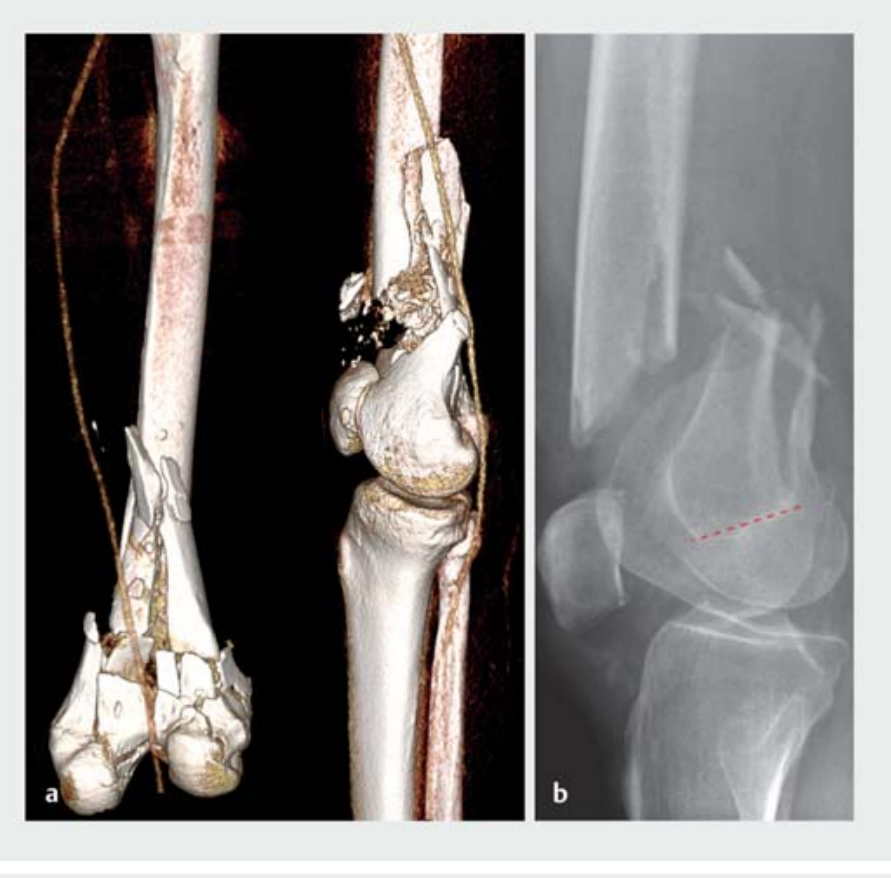

- Abb. 2 a 3-D-Rekonstruktion einer CT-Angiografie einer 46-jährigen Patientin mit distaler Femurfraktur. Man beachte die topografische Nähe der knöchernen Frakturfragmente zur A. poplitea. b Darstellung der Blumensaat-Linie, welche die knöcherne Begrenzung der Notch anzeigt.

Die Computertomografie ist bei intraartikulären distalen Femurfrakturen obligat, um die gesamte Frakturmorphologie vollständig verstehen zu können. Zudem können die koronar verlaufenden Hoffa-Frakturen der Femurkondylen im konventionellen Röntgen übersehen werden [17].

Die A. femoralis zieht im Bereich des Oberschenkels im Adduktorenkanal nach distal und tritt durch den Hiatus adductorius als A. poplitea in die Kniekehle, in der sie weiter durch die Soleusarkade verläuft. Dadurch kann es bei stark dislozierten Frakturen zu Gefäßverletzungen durch scharfkantige Frakturfragmente und/oder Endothelschäden durch Kompression bzw. Zugkräfte kommen ( $\triangleright$ Abb. 2) [8]. Bei der klinischen Untersuchung ist deshalb die Überprüfung der peripheren Durchblutung, Motorik und Sensibilität zwingend notwendig, um neurovaskuläre Begleitverletzungen rasch diagnostizieren zu können. Beim klinischen Verdacht auf eine Gefäßverletzung (expandierendes Hämatom bzw. Weichteilschwellung, fehlende Pulse, persistierende arterielle Blutung bei offenen Verletzungen) ist eine Angiografie indiziert. In den 90er-Jahren galt dabei die digitale Subtraktionsangiografie (DSA = Digital Subtraction Angiography) als Standard [18]. Die DSA ist jedoch durch eine erhöhte Invasivität, eine zeitintensive Vorbereitung und hohe Kosten gekennzeichnet [19]. Diagnostikum der Wahl stellt heutzutage die CT-Angiografie (CTA) dar [18]. Diese ist kosteneffektiver und schneller [20]. Die Genauigkeit bei der Detektion von Gefäßverletzungen an den Extremitäten mittels 
CTA wird mit einer Sensitivität von 96,2\% und einer Spezifität von 99,2\% angegeben [18].

Am Schluss der diagnostischen Kaskade und der abschließenden klinischen Beurteilung steht die Entscheidung zur konservativen oder operativen Behandlung. Die osteosynthetische Stabilisierung kann jedoch in Abhängigkeit von der Weichteilschwellung, Begleitverletzungen bei polytraumatisierten Patienten (z. B. Lungenkontusionen, abdominale Verletzungen, hämorrhagischer Schock), komplexen intraartikulären Frakturen oder offenen Frakturen auch erst sekundär im Verlauf erfolgen. Dann ist zunächst eine Ruhigstellung mittels Schiene, transossärer Extension oder im Fixateur externe indiziert. Insbesondere bei offenen Frakturen, die in 5-10\% der Fälle bei distalen Femurfrakturen auftreten, ist zunächst eine Transfixation mit Wunddébridement und ggf. weiteren programmierten Revisionseingriffen indiziert, um die Gefahr einer periimplantären Infektion nach definitiver Stabilisierung zu verringern $[21,22]$.

\section{Konservative Therapie}

Das konservative Vorgehen bei distalen Femurfrakturen ist ausgewählten Indikationen vorbehalten. Hierzu zählen einfache, nicht dislozierte Frakturen bei Patienten, bei denen von einem Mindestmaß an Compliance ausgegangen werden kann. Ein konservatives Vorgehen kann zudem als temporäre Maßnahme bei Patienten mit erheblichen perioperativen Risiken gewählt werden, bis der Zustand des Patienten die osteosynthetische Stabilisierung zulässt. Prinzipiell lässt sich aber feststellen, dass die konservative Therapie distaler Femurfrakturen durch eine verlängerte Liegedauer sowie Ruhigstellung mit einer erhöhten Komplikationsrate vergesellschaftet ist (Pseudarthrose, Pneumonie, Harnwegsinfekte, tiefe Beinvenenthrombose, Infektionen, funktionelle Einschränkungen durch verlängerte Immobilisation) [23]. Einfache, nicht dislozierte Frakturen können in einer kniegelenksübergreifenden Orthese ruhiggestellt und die Patienten an Unterarmgehstützen mit Entlastung des betroffenen Beins mobilisiert werden. Die Behandlung komplexer distaler Femurfrakturen, bei denen eine Operation aufgrund des Allgemeinzustandes des Patienten kontraindiziert ist, kann bei starker Verkürzung und Dislokation durch eine transossäre Extensionsbehandlung erfolgen. Dabei handelt es sich jedoch ausschließlich um eine temporäre Maßnahme, bis die osteosynthetische Stabilisierung durchgeführt werden kann. Diese Maßnahme ist bei einfachen, nicht dislozierten Frakturen, die rein konservativ behandelt werden, jedoch nicht angezeigt. Hierbei wird im Bereich der proximalen Tibia ein Extensionsdraht in Lokalanästhesie parallel zur Kniegelenksebene eingebracht und die Extremität durch ein angehängtes Gewicht extendiert, um durch axialen Zug an der Fraktur eine längen- und achsgerechte Reposition der Fraktur zu erreichen. Das Extensionsgewicht sollte bei muskelstarken Pa- tienten ein Siebtel des Körpergewichts und bei adipösen/ muskelschwachen Patienten ein Achtel bis ein Zehntel des Körpergewichts betragen. Hierbei ist darauf zu achten, dass der Knick der Braun'schen Schiene des Extensionsbettes nicht in der Kniekehle liegt, sondern proximal davon. Dies soll der posterioren Verkippung der Femurkondylen durch die Fraktur und den Zug des M. gastrocnemius entgegenwirken ( $\bullet$ Abb. 2) [24]. Ziel der konservativen Therapie ist die Wiederherstellung der Beinlänge sowie -achse in der Sagittal- und Koronarebene. Bessert sich der Allgemeinzustand des Patienten, so sollte die Fraktur im Verlauf operiert werden. In Fällen, in denen die Fraktur konservativ ausbehandelt wird, empfiehlt sich der Wechsel auf eine kniegelenksübergreifende Orthese mit freier Beweglichkeit, sobald konventionell-radiologisch eine beginnende knöcherne Konsolidierung gesehen wird und die Weichteilschwellung vollständig zurückgegangen ist. Die Orthese sollte hierbei in Außenrotation und geringer Valgusstellung angebracht werden, um der Fehlstellung der Fraktur entgegenzuwirken [24].

\section{Operative Versorgung}

In den letzten Jahrzehnten galt die offene anatomische Reposition und konventionelle Plattenosteosynthese mit dem Ziel der primären Frakturheilung als akzeptierter Standard, jedoch war diese Art der Frakturversorgung ebenfalls mit einer relativ hohen Rate an Komplikationen (Infektionen, Pseudarthrosen, Achsfehlstellungen, Arthrofibrose) vergesellschaftet $[25,26]$. Durch die Entwicklung winkelstabiler Implantate und neue Erkenntnisse über die Physiologie der Knochenheilung ist es in den letzten 15 Jahren zu einem Paradigmenwechsel gekommen [27-30]. Bei extraartikulären Frakturen gilt als oberstes Ziel nicht mehr die anatomische Reposition, sondern die Wiederherstellung der korrekten Beinlänge und -achse. Die Fraktur wird durch retrograde Nagelosteosynthesen oder winkelstabile Implantate, mit dem Ziel der sekundären Frakturheilung im Sinne eines Fixateur interne, überbrückt [1]. Bei intraartikulären Frakturen ist jedoch nach wie vor die korrekte anatomische Reposition und rigide Fixierung des gelenktragenden Fragments anzustreben [1,29]. Aufgrund der Komplexität der Verletzung sind Osteosynthesen von diesen Frakturen im Tagesprogramm durchzuführen [8]. Zudem ist eine präoperative Planung mit Anfertigung einer Skizze, welche die Frakturmorphologie und Implantatwahl berücksichtigt, empfehlenswert ( $\bullet$ Abb. 3 ).

Ligamentäre Begleitverletzungen wie Rupturen der Kreuz- oder Kollateralbänder bei distalen Femurfrakturen sind selten, fallen in der Primärdiagnostik aufgrund der Schmerzen der Patienten sowie der Weichteilschwellung häufig nur bei knöchernen Avulsionsfrakturen auf. Wenn es zu dieser seltenen Begleitverletzung kommt, ist das vordere Kreuzband (VKB) am häufigsten betroffen [14]. Insbesondere bei intraartikulären Frakturen bzw. bei 


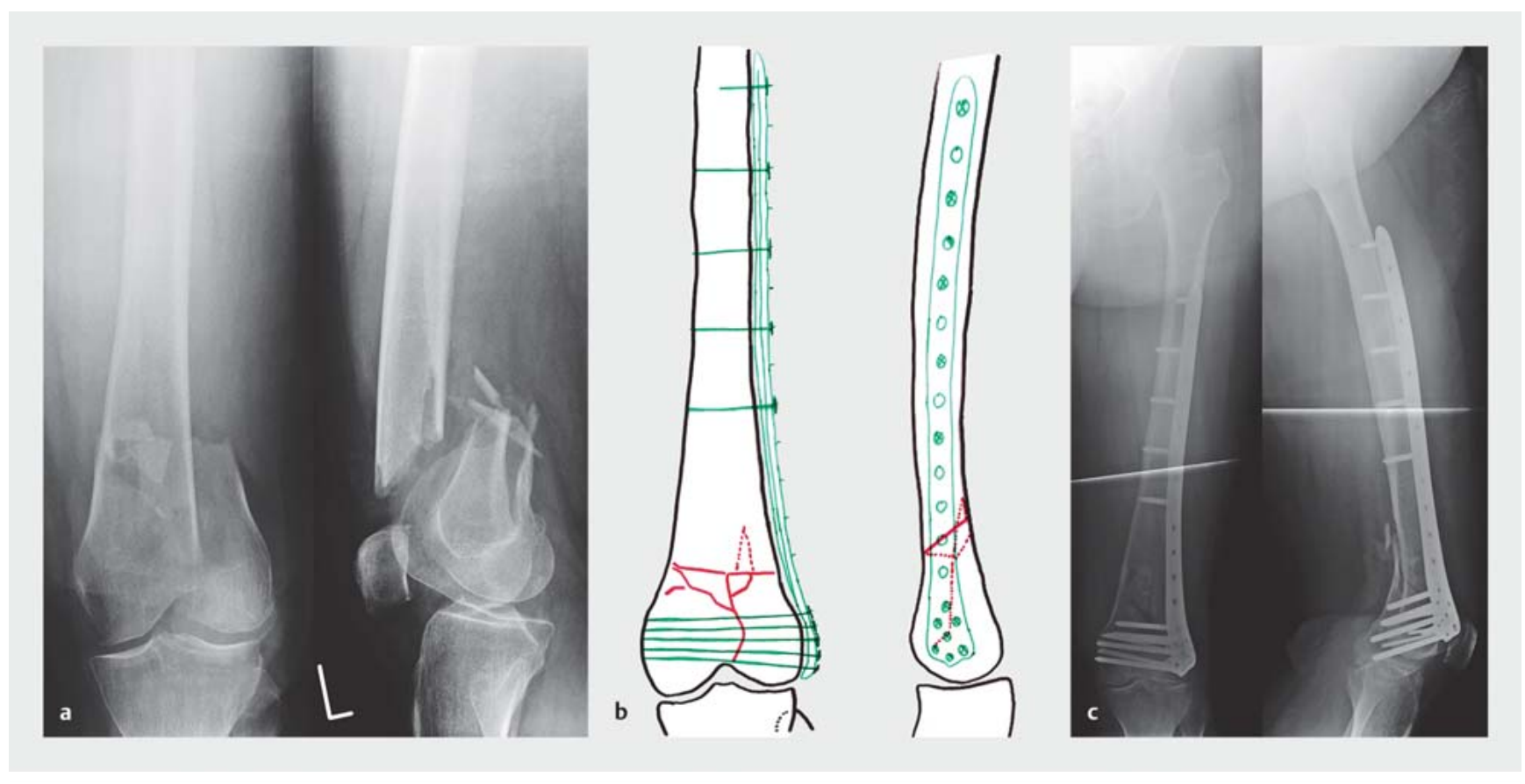

- Abb. 3 Fallbeispiel einer 75-jährigen Patientin nach Sturz auf das linke Bein. a präoperatives Röntgenbild mit typischer Rekurvationstendenz des distalen Frakturfragments nach dorsal, b präoperative Planung mit vorgesehener LISS-Platte, c Röntgenbild 1 Woche postoperativ.

Frakturen, die den knöchernen Ursprung des VKB betreffen, kann es zur Ablösung des Ligaments kommen. Knöcherne Avulsionsfrakturen des VKB können bei der osteosynthetischen Stabilisierung mittels Schraubenosteosynthese mitversorgt werden. Hinsichtlich rein ligamentärer Rupturen gibt es keinen Konsens darüber, ob und wann diese mitzuversorgen sind. Es ist jedoch zu beachten, dass die kombinierte Versorgung durch Osteosynthese und VKB-Plastik zu einer erhöhten Rate an Komplikationen führen kann. Durch das vorhandene Osteosynthesematerial kann das Setzen des Bohrlochs für die Kreuzbandplastik deutlich erschwert werden, was zu einem schlechteren klinischen Ergebnis der VKB-Plastik führen kann [31]. Zudem würde durch diesen zusätzlichen Eingriff die OP-Zeit verlängert und das operative Trauma größer werden, wodurch die Wahrscheinlichkeit einer Infektion und einer Arthrofibrose mit verringerter Range of Motion steigen könnte [8]. Das Setzen des Bohrlochs für die VKB-Plastik kann zudem zu einer sekundären Dislokation von Frakturfragmenten führen. Es wird dementsprechend empfohlen, die Indikation für eine VKB-Plastik sekundär nach der primären Osteosynthese zu stellen [8]. Ligamentäre Rupturen des hinteren Kreuzbandes (HKB) werden in der Regel konservativ durch Ruhigstellung in einer PTS-Schiene (PTS = Posterior Tibial Support) behandelt, knöcherne Ausrisse können ebenfalls mittels Schraubenosteosynthese refixiert werden. Bei Verletzungen der Seitenbänder werden knöcherne Ausrisse ebenfalls bei der definitiven Versorgung durch Schrauben refi- xiert [8]. Rein ligamentäre Verletzungen der Seitenbänder werden in der Regel konservativ behandelt [32].

Bei der Behandlung bzw. Rekonstruktion von Gefäßdefekten mittels temporären Shunts, autologen venösen oder synthetischen Grafts sollte zuvor sichergestellt werden, dass die Fraktur durch einen Fixateur externe zunächst reponiert und ohne Verkürzung fixiert wurde. Dadurch wird verhindert, dass eine zuerst durchgeführte Gefäßrekonstruktion durch darauffolgende Repositionsmanöver Schaden nimmt. Bei Gefäßverletzungen ist zudem immer zu berücksichtigen, dass eine Ischämie nach Reperfusion durch ein Reexpansionsödem zu einem verringerten venösen Rückstrom und entsprechend zu einem Kompartmentsyndrom führen kann. Deshalb sind in diesen Situationen engmaschige klinische Kontrollen obligat, gegebenenfalls kann auch eine prophylaktische Fasziotomie notwendig sein $[8,33]$.

\section{Lagerung}

Die Lagerung des Patienten ist abhängig von der gewählten Osteosynthese. Bei Plattenosteosynthesen wird der Patient in Rückenlage gelagert und eine Knierolle dorsal des distalen Frakturfragments positioniert, um der Rekurvationstendenz der Fraktur entgegenwirken und um bei der Reposition zu helfen ( $\bullet$ Abb. 4). Die Reposition kann zudem erleichtert werden, indem das Kniegelenk leicht gebeugt wird. Hierdurch wird die Spannung vom M. gastrocnemius genommen, der u.a. für die Dislokation der 

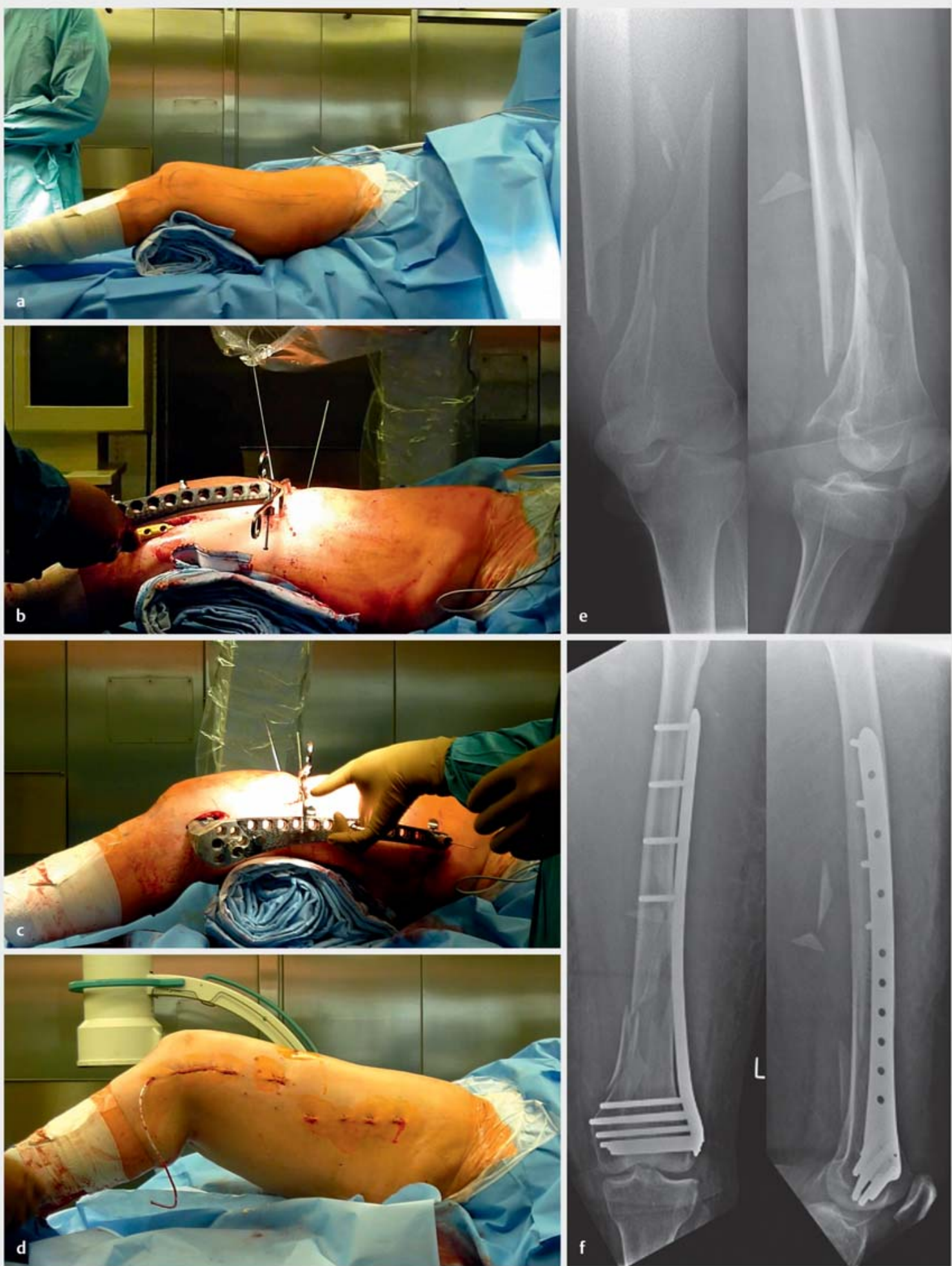

- Abb. 4 Operatives Vorgehen bei minimalinvasiver Platteosteosynthese mittels des Less Invasive Stabilization System (LISS, Fa. DePuy Synthes). a Lagerung des Patienten in Rückenlage mit angehobenem ipsilateralen Gesäß, um das Bein in $0^{\circ}$ Neutralrotation zu halten. Zudem wird ein steriler Kittel unterhalb des Frakturfragments geschoben, um der Rekurvation des distalen Femurs entgegenzuwirken. b Nach lateralem Zugang zur lateralen Femurepikondyle wird die Platte zwischen Knochen und M. vastus lateralis weichteilschonend geschoben. $\mathbf{c}$ Besetzen der proximalen Schrauben. $\mathbf{d}$ Wundverschluss. e präoperativer röntgenologischer Befund. $f$ postoperativer röntgenologischer Befund. 
Fraktur verantwortlich ist (siehe Punkt Anatomie) [34]. Das kontralaterale Bein wird abgesenkt, um seitliche Kontrollen durch den BV zu vereinfachen. Bei Nagelosteosynthesen kann mit verschiedenen Hilfen, z. B. Dreiecksstütze oder Knierolle, das Knie $70-90^{\circ}$ gebeugt werden, um die Nagelimplantation zu vereinfachen. Eine Blutsperre ist in beiden Fällen nicht notwendig.

\section{Zugänge}

\section{Lateraler Zugang}

Der laterale Zugang ist prinzipiell der Standardzugang für die offene Reposition und Plattenosteosynthese distaler Femurfrakturen. Die Hautinzision erfolgt hier über der lateralen Femurepikondyle, und wird nach proximal je nach Frakturmorphologie und präoperativer Planung verlängert. Anschließend wird der Tractus iliotibialis entlang des Faserverlaufs gespalten. Unter dem Tractus iliotibialis liegen zahlreiche Äste der A. genus superior lateralis mit begleitenden Venen, welche ligiert werden müssen. Der darunter liegende M. vastus lateralis wird stumpf und weichteilschonend nach anterior mobilisiert. Der Zugang erlaubt eine gute Sicht auf das laterale Femur und die laterale Femurepikondyle [35].

\section{Anterolateraler Zugang}

Der anterolaterale Zugang ist eine Modifikation des lateralen Zugangs und erlaubt eine bessere Darstellung der Gelenkfläche für die Reposition von intraartikulären Frakturen. Distal wird die Haut ca. 6-8 cm longitudinal über dem anterioren Aspekt der lateralen Femurepikondyle inzidiert und verlängert sich nach proximal entsprechend dem gewählten Implantat und der Fraktur. Nach der Hautinzision distal erfolgt die Präparation durch das subkutane Fettgewebe bis auf den Tractus iliotibialis und das Retinaculum patellae laterale. Der Tractus iliotibialis wird entsprechend des lateralen Zugangs im Faserverlauf gespalten, die darunter liegenden Äste der A. und V. genus superior lateralis ligiert und der M. vastus lateralis stumpf nach anterior mobilisiert, um ein Plattenlager auf dem lateralen Femur zu schaffen. Im Bereich des Kniegelenks wird das Retinaculum patellae laterale scharf gespalten, um Sicht auf die Kniegelenkskapsel zu erhalten. Diese wird ebenfalls longitudinal gespalten und die Patella nach medial luxiert, um eine gute Sicht auf die Gelenkfläche zu bekommen $[8,35]$.

\section{Minimalinvasiver Zugang}

Für eingeschobene Platten im Sinne einer minimalinvasiven Plattenosteosynthese (MIPO = minimally invasive plate osteosynthesis) kann sowohl der laterale als auch der anterolaterale Zugang modifiziert werden. Dabei wird die Hautinzision und Präparation nicht nach proximal fortgesetzt, sondern proximal - in Verlängerung der Platte und im Bereich der dort vorgesehenen Schrauben - eine zusätzliche Inzision gemacht. Dadurch wird das operative Weichteiltrauma reduziert, da der M. vastus lateralis nicht über eine längere Strecke vom Femur abgelöst wird [35].

\section{Minimalinvasiver medialer parapatellarer Zu- gang für retrograde Nagelosteosynthesen}

Der Patient wird hierbei ebenfalls in Rücklage gelagert, das Kniegelenk ist dabei jedoch $90^{\circ}$ gebeugt. Zudem ist auf die korrekte Rotation des Beins zu achten. Unter die ipsilaterale Gesäßhälfte sollte ein Polster geschoben werden, um das Bein in $0^{\circ}$ Rotationsstellung zu halten - die Patella liegt dabei direkt obenauf. Der Hautschnitt erfolgt im Bereich der Patellarsehne $1 \mathrm{~cm}$ medial der medialen Begrenzung der Patella und $2 \mathrm{~cm}$ unterhalb des distalen Patellapols und wird für $3 \mathrm{~cm}$ nach distal verlängert. Unter Ausnutzung des Hautschnittes erfolgt die subkutane Präparation bis auf das Retinaculum patellae, welches medial der Patellarsehne inzidiert wird. Anschließend wird die Kniegelenkskapsel dargestellt und ebenfalls longitudinal durchtrennt. Dadurch erhält man Sicht auf das Kniegelenk mit der interkondylären Notch [35]. Der femorale Ansatzpunkt des hinteren Kreuzbandes befindet sich im lateralen Bereich der medialen Femurkondyle. Um diesen Bereich durch die Nagelimplantation zu schonen, befindet sich der Eintrittspunkt des Nagels in Verlängerung der anatomischen Femurachse im Bereich der interkondylären Notch ca. $1 \mathrm{~cm}$ anterior und lateral des femoralen Insertionsbereichs des HKB [36].

Cave
Im distalen Bereich der Inzision befindet sich der in-
frapatelläre Ast des sensiblen N. saphenus [35].

\section{Medialer Zugang}

Der mediale Zugang findet vor allem für koronare B-Verletzungen, also isolierte Frakturen der medialen Femurkondyle (Hoffa-Frakturen), Verwendung. Die Hautinzision beginnt über dem Tuberculum adductorium und wird nach proximal posterior des $\mathrm{M}$. vastus medialis fortgesetzt. Die Faszie des M. vastus medialis wird in Faserrichtung gespalten und der Muskel nach anterior mobilisiert. Anschließend erfolgt eine quer nach anterior verlaufende Tenotomie der Vastus-medialis-Sehne, die L-förmig longitudinal nach distal im Sinne einer parapatellaren Arthrotomie verlängert wird [8].

\footnotetext{
Cave

Beim medialen Zugang ist bei der proximalen Verlängerung auf den Hiatus adductorius zu achten, durch den die Gefäß-Nerven-Straße zieht.
}

\section{Implantate}

Grundsätzlich stehen für die Versorgung distaler Femurfrakturen diverse Schrauben, Platten und Osteosynthesenägel zur Verfügung, die entsprechend der Frakturmorphologie und -klassifizierung eingesetzt werden können. 


\section{Winkelstabile Platten}

Winkelstabile Plattenosteosynthesen kommen bei distalen Femurfrakturen vor allem bei extraartikulären Aund intraartikulären C-Verletzungen zur Verwendung. Auf dem Markt zur Verfügung stehende Implantate sind zum einen monoaxiale winkelstabile Platten wie das Less invasive Stabilization System for Distal Femur (LISS-DF, Fa. DePuy Synthes), die PERI-LOC distale Femurplatte (Fa. Smith \& Nephew) oder die LOQTEQ Distale Laterale Femurplatte (Fa. aap Implantate). Ebenfalls verfügbar sind auch polyaxiale winkelstabile Implantate wie die variable Angle-Locking Compression Plate (VA-LCP-) Kondylenplatte (Fa. DePuy Synthes) oder die Non-Contact Bridging Plate for Distal Femur (NCB-DF, Fa. Zimmer) [36].

\section{Retrograde Marknagelosteosynthesen}

Retrograde Marknagelosteosynthesen sind bei distalen Femurfrakturen insbesondere bei extraartikulären, suprakondylären Femurfrakturen indiziert. Marknägel können im Vergleich zu den Plattenosteosynthesen, solange die Frakturmorphologie und -lokalisation dies erlauben, eine biomechanisch stabilere Frakturversorgung gewährleisten. Nachteil von retrograd eingebrachten Nägeln ist jedoch, dass das Kniegelenk hierfür eröffnet werden muss. Besonders bei jungen Patienten ist deshalb auf den Schutz des Knorpels und der Knie-Binnenstrukturen zu achten. Aktuell auf dem Markt verfügbare Implantate sind z. B. der Distal Femoral Nail (DFN, Fa. DePuy Synthes), der T2 Supracondylar Nail (Fa. Stryker) oder der TRIGEN IM Knee Nail (Fa. Smith \& Nephew) $[8,36]$.

\section{Vorgehen nach Frakturmorphologie}

\section{Typ-A-Verletzungen}

Extraartikuläre distale und suprakondyläre Femurfrakturen können prinzipiell mittels winkelstabiler Plattenosteosynthesen oder retrograder Verriegelungsnägeln versorgt werden. Aus biomechanischer Sicht ist die Verwendung von Nagelosteosynthesen Plattenosteosynthesen zu bevorzugen, jedoch richtet sich die Entscheidung nach weiteren Kriterien wie dem Alter der Patienten, der Knochenqualität, einer ggf. vorhandenen Hüft-Totalendoprothese oder weiteren Begleitverletzungen wie z.B. einer Patellafraktur [8].

\section{Typ-B-Verletzungen}

B-Frakturen bzw. partiell intraartikuläre Frakturen sind isolierte uni- bzw. bikondyläre Frakturen der Femurkondylen, die sagittal (B1-B2) oder koronar (B3), wie im Falle einer Hoffa-Fraktur, verlaufen. Diese Frakturen können prinzipiell über eine Zugschraubenosteosynthese stabilisiert werden. In einigen Fällen, wie bei größeren Frakturfragmenten, bietet es sich an, die Osteosynthese über eine zusätzliche Abstützplatte zu sichern [8].

\section{Typ C-Verletzungen}

Bei intraartikulären Frakturen ist bei der operativen Versorgung zunächst die Arthrotomie und offene Reposition primäres Ziel. Nach Stellen des distalen Gelenkblocks kann die achs- und rotationsgerechte Osteosynthese erfolgen. Dies wird primär mittels winkelstabiler Plattenosteosynthesen durchgeführt, kann bei C1-C2-Frakturen aber auch mittels retrograder Verriegelungsnägeln erfolgen [8]. Bei C-Verletzungen mit einfachen Frakturverläufen kann es für die Reposition und Stabilität hilfreich sein, zunächst eine interfragmentäre Zugschraube zu setzen [37]. Dies widerspricht zwar der etablierten Auffassung der winkelstabilen Plattenosteosynthese als „Fixateur interne“, die klinischen Daten zeigen jedoch, dass eine zusätzliche Zugschraube in Kombination mit einer Plattenosteosynthese der alleinigen Plattenosteosynthese überlegen sein kann [38].

\section{Hinweise für das operative Vorgehen}

- Ziel der Reposition ist es, der durch muskuläre Kräfte entstehenden Innenrotations-, Verkürzungs- und Varusfehlstellung entgegenzuwirken. Der M. gastrocnemius verkippt durch seinen muskulären Ursprung das distale Frakturfragment nach dorsal, während der M. adductor magnus durch seinen Ansatz am Tuberculum adductorium des medialen Epicondylus die Fraktur in eine Innenrotations- und Varusfehlstellung bringt. Um die Reposition zu erleichtern, wird eine Rolle dorsal des distalen Femurs positioniert, um die Rekurvation auszugleichen. Weiter kann der Verkippung durch den M. gastrocnemius entgegengewirkt werden, indem das Kniegelenk leicht gebeugt wird [34] ( Abb. 4).

- Zusätzlich kann die Reposition auch durch transkutan eingebrachte Schanz'sche Schrauben in das Frakturfragment erfolgen, die dann als „Joystick“ verwendet werden [34].

- Bei Frakturen, bei denen eine geschlossene Reposition nicht adäquat möglich ist, sollte eine offene Reposition erfolgen [34].

- Die mechanische Beinachse kann überprüft werden, indem ein Diathermie-Stromkabel vom Hüftkopfzentrum bis zum Zentrum des oberen Sprunggelenks entsprechend der Mikulicz-Linie gelegt wird und die Achse durch Röntgenbilder kontrolliert wird. Im Bereich des Kniegelenks verläuft die mechanische Achse physiologisch jedoch nicht zentral durch das Gelenk, sondern trifft das femorotibiale Kompartiment ca. 5$10 \mathrm{~mm}$ medial des Kniegelenkzentrums (siehe Abschnitt Anatomie).

- Beim Einbringen des Marknagels ist zudem auf regelmäßige Röntgenkontrollen und einen ausreichenden axialen Zug am Bein durch den Assistenten zu achten, um Verkürzungen oder Achsfehlstellungen zu vermeiden und rechtzeitig zu erkennen.

- Um eine Malrotation nach Reposition zu verifizieren, kann das Trochanter-minor-Zeichen verwendet wer- 
den. Dabei wird mittels Bildwandlerkontrolle das kontralaterale Bein in die Neutralrotation gebracht, d.h. die Patella zeigt nach ventral. Dann wird ein Röntgenbild von der Hüfte gemacht und gespeichert. Die gleiche Prozedur wird nun auf der betroffenen Beinseite wiederholt und die Röntgenbilder beider Hüften verglichen. Eine korrekte Rotation ist erreicht, wenn der Trochanter minor auf beiden Seiten die gleiche Form hat. Erscheint der Trochanter minor auf der betroffenen Seite kleiner, besteht eine fehlerhafte Außenrotation, da ein Teil vom Femurschaft überstrahlt wird. Bei einer fehlerhaften Innenrotation erscheint der Trochanter minor auf der betroffenen Seite größer [34].

- Bei der Überprüfung der korrekten Schraubenlage im Bereich des distalen Femurs ist zum einen auf die Blumensaat'sche Linie zu achten ( $\triangleright$ Abb.2), um Fehllagen in der interkondylären Notch zu vermeiden [7, 34]. Weiterhin ist bei von lateral eingebrachten Schrauben das Bein im a.-p. Röntgen nach außen zu rotieren, um die ventrale und dorsale Kortikalis des Femurs aufgrund der trapezoiden Form übereinander zu projizieren ( $\bullet$ Abb. 1, siehe Punkt Anatomie) [34].

\section{Nachbehandlung}

Die postoperative Mobilisation sollte, soweit möglich, an Unterarmgehstützen mit Tippbelastung des betroffenen Beins für ca. 6-12 Wochen durchgeführt werden [8]. Es kann keine generelle Empfehlung gegeben werden, wann der Übergang in den schrittweisen Belastungsaufbau stattfinden kann. Entscheidend ist dabei die Frakturmorphologie. Frakturen mit metaphysärer Trümmerzone, die anatomisch nicht reponiert wurden und durch sekundäre Knochenheilung heilen sollen, weisen eine längere Heilungsphase auf als isolierte Kondylenfrakturen, die anatomisch reponiert wurden und durch primäre Knochenheilung heilen [8].

Die Ruhigstellung in einer fixierten Kniegelenksorthese mit leichter Beugung sollte bis zur Wundheilung bzw. Weichteilkonsolidierung erfolgen. Anschließend erfolgt der Wechsel auf eine achsgeführte Kniegelenksorthese mit freier Beweglichkeit [8]. Um einer Arthrofibrose, vor allem bei intraartikulären Frakturen und Zugängen mit Arthrotomie, vorzubeugen, wird eine frühfunktionelle Beübung des Kniegelenks durchgeführt. Die postoperative Nachbehandlung mittels CPM-Schiene (Continuous Passive Motion, Motorschiene) wird bei distalen Femurfrakturen kontrovers diskutiert [8]. Hill et al. konnten in einer prospektiven randomisierten Studie keinen signifikanten Unterschied in der Range of Motion nach 6 Monaten bei Patienten feststellen, die mit oder ohne CPMSchiene nachbehandelt wurden [39]. Eine klare Nachbehandlungsempfehlung kann aber aufgrund der geringen Studienzahl mit entsprechend fehlender Evidenz nicht ausgesprochen werden. Bei offenen Frakturen mit Verletzung und operativer Versorgung des Streckappa- rats kann zudem eine Ruhigstellung des Kniegelenks in $0^{\circ}$ für bis zu 6 Wochen notwendig sein [40]. Im eigenen Vorgehen streben wir bei diesen Verletzungen grundsätzlich eine übungsstabile Versorgung an und beginnen in der Regel am 2. postoperativen Tag mit der frühfunktionellen Nachbehandlung.

\section{Komplikationen}

Distale Femurfrakturen müssen, auch wenn es die Frakturmorphologie auf den ersten Blick anders erscheinen lässt, immer als komplexe Verletzung gesehen werden. Dies betrifft nicht nur die chirurgische Technik, die aufgrund minimalinvasiver Zugänge erfahrenen Operateuren vorbehalten sein sollte, sondern auch das perioperative Management. Die Komplikationsrate bei der Versorgung distaler Femurfrakturen wird in der Literatur mit 13,8\% angegeben. Märdian et al. konnten hierbei zeigen, dass insbesondere chirurgische Einflussfaktoren wie der Weichteilschaden, die Frakturklassifikation, das Verletzungsmuster oder der OP-Zeitpunkt zu einer signifikant erhöhten Komplikationsrate führten [1]. Prozentuale Angaben zur Häufigkeit dieser Komplikationen werden in der Literatur für Pseudarthrosen zwischen 5,1-8,5\%, für mechanische Implantatversagen zwischen 1,5-4,9\%, für Pneumonien mit $10,1 \%$ und für Wundinfektionen zwischen 0,4-7,6\% angegeben. Die Revisionsrate liegt je nach Literaturangabe zwischen 12,7 und 30,6\% [1,41].

\section{Fazit}

Distale Femurfrakturen sind komplexe Verletzungen, die stets mit weiteren Verletzungen und Komorbiditäten vergesellschaftet sein können und trotz moderner OP-Verfahren komplikationsbehaftet sind. Neben der erforderlichen Infrastruktur, um polytraumatisierte Patienten oder geriatrische Patienten adäquat perioperativ zu versorgen, sind diese Frakturen nur von erfahrenen Operateuren im Tagesprogramm primär zu stabilisieren. Die Wahl des Implantats und des operativen Zugangs sind abhängig von der Frakturmorphologie und -klassifikation. Hierbei haben sich vor allem minimalinvasive, eingeschobene Plattenosteosynthesen durchgesetzt, die über einen lateralen Zugang eingebracht werden.

\section{Interessenkonflikt}

Die Autoren geben an, dass kein Interessenkonflikt besteht.

\section{Autorinnen/Autoren}

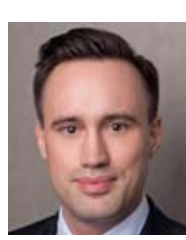

\section{Frank Graef}

Dr. med., Assistenzarzt, Centrum für Muskuloskeletale Chirurgie, Charité-Universitätsmedizin Berlin 


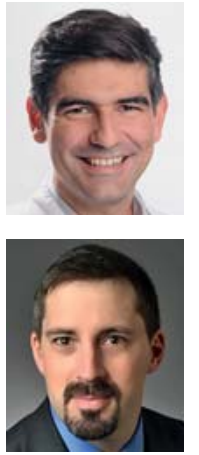

\section{Serafim Tsitsilonis}

Dr. med., Oberarzt, Centrum für Muskuloskeletale Chirurgie, Charité-Universitätsmedizin Berlin

\section{Sven Märdian}

Priv.-Doz. Dr. med., Kommissarischer Geschäftsführender Direktor, Centrum für Muskuloskeletale Chirurgie, Charité-Universitätsmedizin Berlin

Korrespondenzadresse

Dr. med. Frank Graef

Charité - Universitätsmedizin Berlin

Centrum für Muskuloskeletale Chirurgie

Augustenburger Platz 1

13353 Berlin

frank.graef@charite.de

\section{Literatur}

[1] Märdian S, Rau D, Schwabe P et al. Operative Therapie von distalen Femurfrakturen. Orthopade 2016; 45: 32-37

[2] Heim UF. [Defining the boundary between diaphysis and metaphysis using quadrant measurement. A contribution to the classification and documentation of fractures of long tubular bones exemplified by the distal tibia]. Unfallchirurg 1987; 90 : 274-280

[3] Schatzker J, Lambert DC. Supracondylar fractures of the femur. Clin Orthop Relat Res 1979; (138): 77-83

[4] Elsoe R, Ceccotti AA, Larsen P. Population-based epidemiology and incidence of distal femur fractures. Int Orthop 2018; 42: 191-196

[5] Court-Brown CM, Caesar B. Epidemiology of adult fractures: A review. Injury 2006; 37: 691-697

[6] Ehlinger M, Ducrot G, Adam P et al. Distal femur fractures. Surgical techniques and a review of the literature. Orthop Traumatol Surg Res 2013; 99: 353-360

[7] Blumensaat C. Die Lageabweichungen und Verrenkungen der Kniescheibe. Ergebn Chir Orthop 1938; 31: 149-223

[8] Collinge CA, Wiss DA. Distal Femur Fractures. In: Court-Brown CM, Heckman JD, McQueen MM, Ricci WM, Tornetta P, McKee MD, eds. Rockwood and Green's Fractures in Adults. Philadelphia: Lippincott Williams \& Wilkins; 2014

[9] Butt WP, Samuel E. Radiologic anatomy of the proximal end of the femur. J Can Assoc Radiol 1966; 17: 103-106

[10] Luo CF. Reference axes for reconstruction of the knee. Knee 2004; 11: 251-257

[11] Hsu RW, Himeno S, Coventry MB et al. Normal Axial Alignment of the Lower Extremity and Load-Bearing Distribution at the Knee. Clin Orthop Relat Res 1990; 255: 215-227

[12] Matsuda S, Miura H, Nagamine R et al. Posterior tibial slope in the normal and varus knee. Am J Knee Surg 1999; 12: 165-168

[13] Schünke M, Schulte E, Schumacher U. Prometheus LernAtlas der Anatomie. Allgemeine Anatomie und Bewegungssystem. Stuttgart: Thieme; 2014
[14] Bertin KC, Goble EM. Ligament injuries associated with physeal fractures about the knee. Clin Orthop Relat Res 1983; (177): 188-195

[15] Muñoz Vives J, Bel J-C, Capel Agundez A et al. The floating knee: a review on ipsilateral femoral and tibial fractures. EFORT Open Rev 2017; 1: 375-382

[16] Emami Meybodi MK, Ladani MJ, Emami Meybodi T et al. Concomitant ligamentous and meniscal knee injuries in femoral shaft fracture. J Orthop Traumatol 2014; 15: 35-39

[17] Nork SE, Segina DN, Aflatoon K et al. The association between supracondylar-intercondylar distal femoral fractures and coronal plane fractures. J Bone Joint Surg Am 2005; 87: 564-569

[18] Jens S, Kerstens MK, Legemate DA et al. Diagnostic Performance of Computed Tomography Angiography in Peripheral Arterial Injury due to Trauma: A Systematic Review and Metaanalysis. Eur J Vasc Endovasc Surg 2013; 46: 329-337

[19] Patterson BO, Holt PJ, Cleanthis M et al.; London Vascular Injuries Working Group. Imaging vascular trauma. Br J Surg 2012; 99: 494-505

[20] Wallin D, Yaghoubian A, Rosing D et al. Computed Tomographic Angiography as the Primary Diagnostic Modality in Penetrating Lower Extremity Vascular Injuries: A Level I Trauma Experience. Ann Vasc Surg 2011; 25: 620-623

[21] Weight M, Collinge C. Early results of the less invasive stabilization system for mechanically unstable fractures of the distal femur (AO/OTA types A2, A3, C2, and C3). J Orthop Trauma 2004; 18: 503-508

[22] Dugan TR, Hubert MG, Siska PA et al. Open supracondylar femur fractures with bone loss in the polytraumatized patient Timing is everything! Injury 2013; 44: 1826-1831

[23] Butt MS, Krikler S], Ali MS. Displaced fractures of the distal femur in elderly patients. Operative versus non-operative treatment. J Bone Joint Surg Br 1996; 78: 110-114

[24] Jahna H, Wittich H. Konservative Methoden in der Frakturbehandlung. München: Urban \& Schwarzenberg; 1985

[25] Moore T], Watson T, Green SA et al. Complications of surgically treated supracondylar fractures of the femur. J Trauma 1987; 27: 402-406

[26] Merchan EC, Maestu PR, Blanco RP. Blade-plating of closed displaced supracondylar fractures of the distal femur with the AO system. J Trauma 1992; 32: 174-178

[27] Kayali C, Agus H, Turgut A. Successful results of minimally invasive surgery for comminuted supracondylar femoral fractures with LISS: comparative study of multiply injured and isolated femoral fractures. J Orthop Sci 2007; 12: 458-465

[28] Märdian S, Schaser KD, Duda GN et al. Working length of locking plates determines interfragmentary movement in distal femur fractures under physiological loading. Clin Biomech (Bristol, Avon) 2015; 30: 391-396

[29] Perren SM. Evolution of the internal fixation of long bone fractures. The scientific basis of biological internal fixation: choosing a new balance between stability and biology. J Bone Joint Surg Br 2002; 84: 1093-1110

[30] Augat P, von Rüden C. Evolution of fracture treatment with bone plates. Injury 2018; 49: S2-S7

[31] Forsythe B, Kopf S, Wong AK et al. The location of femoral and tibial tunnels in anatomic double-bundle anterior cruciate ligament reconstruction analyzed by three-dimensional computed tomography models. J Bone Joint Surg Am 2010; 92: 1418-1426

[32] Kramer DE, Miller PE, Berrahou IK et al. Collateral Ligament Knee Injuries in Pediatric and Adolescent Athletes. J Pediatr Orthop 2017. doi:10.1097/BPO.0000000000001112 
[33] Farber A, Tan TW, Hamburg NM et al. Early fasciotomy in patients with extremity vascular injury is associated with decreased risk of adverse limb outcomes: A review of the National Trauma Data Bank. Injury 2012; 43: 1486-1491

[34] Buckley R, Moran C, Apivatthakakul T. AO Principles of Fracture Management. Stuttgart: Thieme; 2018

[35] Hoppenfeld S, de Boer P, Buckley R. Surgical Exposures in Orthopaedics. The anatomic Approach. 5th ed. Alphen aan den Rijn: Wolters Kluwer; 2017

[36] Bliemel C, Bücking B, Ruchholtz S. Distale Femurfrakturen. Orthopädie und Unfallchirurgie up2date 2017; 12: 63-84

[37] Märdian S, Schmölz W, Schaser KD et al. Interfragmentary lag screw fixation in locking plate constructs increases stiffness in simple fracture patterns. Clin Biomech (Bristol, Avon) 2015; 30: $814-819$

[38] Chung JY, Cho JH, Kweon HJ et al. The use of interfragmentary positional screw in minimally invasive plate osteosynthesis for simple distal femur fractures in elderly patients: A retrospective, single-centre pilot study. Injury 2016; 47: 2795-2799
[39] Hill AD, Palmer M], Tanner SL et al. Use of Continuous Passive Motion in the Postoperative Treatment of Intra-Articular Knee Fractures. J Bone Joint Surg Am 2014; 96: e118

[40] Langenhan R, Baumann M, Ricart P et al. Postoperative functional rehabilitation after repair of quadriceps tendon ruptures: a comparison of two different protocols. Knee Surg Sports Traumatol Arthrosc 2012; 20: 2275-2278

[41] Zlowodzki M, Bhandari M, Marek DJ et al. Operative treatment of acute distal femur fractures: systematic review of 2 comparative studies and 45 case series (1989 to 2005). J Orthop Trauma 2006; 20: 366-371

Bibliografie

DOI https://doi.org/10.1055/a-0755-5681

Online-publiziert 15.01.2019 | OP-JOURNAL 2019; 34: 145155 @ Georg Thieme Verlag KG Stuttgart · New York ISSN 0178-1715 